\title{
Déguisement et théâtralité
}

\section{Henri Suhamy}

\section{(2) OpenEdition \\ 1 Journals}

Édition électronique

URL : http://journals.openedition.org/shakespeare/1477

DOI : $10.4000 /$ shakespeare. 1477

ISSN : 2271-6424

Éditeur

Société Française Shakespeare

\section{Édition imprimée}

Date de publication : 20 décembre 2008

Pagination : 161-176

ISBN : 2-9521475-5-8

\section{Référence électronique}

Henri Suhamy, "Déguisement et théâtralité », Actes des congrès de la Société française Shakespeare [En ligne], 26 | 2008, mis en ligne le 20 décembre 2008, consulté le 19 avril 2019. URL : http:// journals.openedition.org/shakespeare/1477; DOI : 10.4000/shakespeare.1477 


\section{Costume et déguisement dans le théâtre de Shakespeare et de ses contemporains}

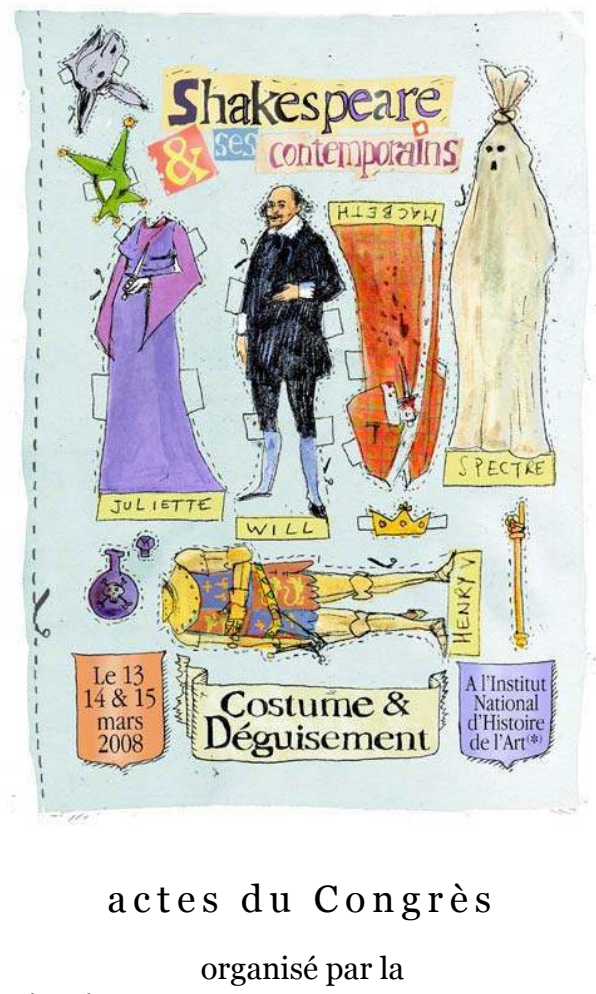

SOCIÉTÉ FRANÇAISE SHAKESPEARE

les 13,14 et 15 mars 2008

textes réunis par

Pierre KAPITANIAK

sous la direction de

Jean-Michel DÉPRATS 
COUVERTURE :

Edouard Lekston,

William en carton et ses habits à découper, 2008 edlek@free.fr

conception graphique et logo

Pierre Kapitaniak

\section{(C) 2008 Société Française Shakespeare}

Institut du Monde Anglophone

Université de Paris III - Sorbonne Nouvelle

5 rue de l'École de Médecine

75006 Paris

www.societefrancaiseshakespeare.org

Tous droits de traduction, de reproduction et d'adaptation réservés pour tous les pays 


\title{
DÉGUISEMENT ET THÉÂTRALITÉ
}

\author{
Henri SUHAMY
}

\begin{abstract}
Le théâtre, particulièrement celui de Shakespeare, est le lieu privilégié du déguisement, qui se manifeste sous deux formes principales, reposant sur une connivence entre l'auteur et le public. D'une part les acteurs assument au cours de leur carrière un grand nombre de personnalités différentes, d'autre part les personnages eux-mêmes sont souvent amenés par l'intrigue à adopter un déguisement. Toutes les comédies de Shakespeare utilisent le procédé, à l'exception de The Comedy of Errors où la gémellité suffit à provoquer les quiproquos. La pratique du déguisement est liée aussi aux thèmes du mensonge, du faux-semblant, de la tromperie, mais le discours moralisateur qui en découle contient aussi une réflexion sur la théâtralité de la vie elle-même, où chacun assume un certain nombre de rôles et de masques.
\end{abstract}

The theatre, especially in Shakespeare's works for the stage, is the right place for disguise, which appears in two main forms, both relying on a close connivance between the author and the public. On the one hand all players, throughout their careers, take on a great number of different personalities; on the other hand, the characters themselves are often brought by the plot to adopt disguises. The device is used by Shakespeare in all his comedies, except The Comedy of Errors, in which resemblance between twins suffices to cause comic misunderstandings. The practice of disguise is also connected with the themes of lies, false appearances and deceit but the moralizing discourse deriving from these themes also contains an underlying meditation on the theatrical aspects of life itself, in which everyone assumes a certain number of roles and masks.

$\mathrm{L}$ e théâtre, celui de Shakespeare en particulier, est le lieu privilégié du déguisement, procédé qui se présente sous deux formes et emplit deux fonctions différentes, quoique forcément liées l'une à l'autre. Chacune de ces deux formes et de ces deux fonctions entraîne des subdivisions, qui apportent quelque diversité tout en se rattachant à un concept unificateur. Dans les deux cas le procédé apparaît comme spécifiquement théâtral et repose sur une connivence étroite entre l'auteur et le public, connivence plus agissante que la simple acceptation passive par ce dernier d'une convention indispensable au fonctionnement de l'art dramatique. Pour sacrifier à la mode du vocabulaire savant, on peut désigner le premier type de déguisement comme étant extra-diégétique, et le second comme intradiégétique. Le premier type concerne tout le personnel de l'institution théâtrale, le second émane en premier lieu de l'auteur, qui en indique les données dans le texte et dans les didascalies, pour ensuite se matérialiser sur la scène.

Commençons par le premier type. Les acteurs, tous, non uniquement les garçons travestis en femmes selon les habitudes et les règlements auxquels il fallait jadis se conformer, revêtent un déguisement avant d'entrer en scène, même si cette transformation ne 
prend pas un aspect vestimentaire, ou ne se traduit pas ostensiblement par une grosse couche de maquillage, accessoire moins lourdement souligné de nos jours que naguère, mais non complètement abandonné, notamment quand il présente des aspects clownesques. De même les acteurs du temps de Shakespeare ne portaient pas de masques sur la scène, contrairement à ce qui se passait dans l'Antiquité, mais cela n'empêchait pas un certain type de spectacle, cultivé surtout sous les premier Stuarts, de s'appeler précisément des masques. Quoi qu'il en soit des fluctuations dans l'histoire du théâtre, la question vestimentaire n'est jamais négligeable, notamment sur les scènes d'aujourd'hui, où l'on assiste souvent à des décalages voulus entre le contexte référentiel de la pièce et l'habillage des acteurs et des actrices. Il existe aussi une tradition selon laquelle les costumiers donnent libre cours à leur imagination et créent des vêtements qui n'ont jamais existé ailleurs que sur la scène d'un théâtre. Ces costumes imaginaires peuvent être suscités, en vertu d'un certain symbolisme, par le texte même de la pièce. En pratiquant cette autarcie vestimentaire l'art théâtral rejoint l'art chorégraphique, lequel paradoxalement use d'accoutrements aussi fonctionnels que les tenues des gymnastes, et pourtant aussi fantastiques que ceux qui proviennent du pinceau des peintres les plus oniriques. Le public approuve ou non, mais dans tous les cas il prend conscience de l'effet de double déguisement que la régie a voulu provoquer et il y participe à sa manière par le seul fait de prendre conscience des décalages ainsi produits et d'en tirer des réflexions d'ordre esthétique.

D'une façon qu'on peut juger moins superficielle, plus en rapport avec la signification profonde de l'art théâtral, se produit un travestissement psychologique. Tout comédien dans l'exercice de sa profession assume une personnalité autre que la sienne, quelquefois plusieurs personnalités, s’il joue plusieurs rôles au cours du même spectacle. D'ailleurs le goût de la métamorphose et du changement de personnalité fait partie de la vocation des acteurs, dont la carrière constitue une sorte de métempsycose permanente. De nos jours certaines compagnies vont au-delà de la simple nécessité professionnelle et cultivent le travestissement comme une fin en soi plus que comme un moyen, lorsque par exemple acteurs et actrices intervertissent leurs rôles, ou choisissent des rôles ostensiblement à contre-emploi, affichant par exemple un écart visible entre l'âge 
supposé du personnage et celui de l'acteur ou de l'actrice. En tant que régisseur de ses œuvres, et sans oublier le recours imposé aux jeunes garçons dans les rôles féminins, Shakespeare n'allait sans doute pas jusqu'à de telles démonstrations, mais la théâtralité, matérialisée par des artifices non dissimulés, est toujours très assumée chez lui. De plus il tire des réflexions quasi philosophiques des avatars psychologiques et autres, notamment des usurpations sociales auxquelles conduit la profession d'acteur. Dans Ham let par exemple, il laisse entendre que si un acteur est capable de tenir sur la scène le rôle d'un roi, les hommes qui dans la réalité occupent la même position ne sont peut-être fondamentalement que des comédiens à qui l'aveugle Fortune a attribué un emploi.

Il existe cependant des acteurs et des actrices doués d'une personnalité si marquée et si marquante qu'ils l'imposent aux personnages qu'ils incarnent, de sorte que par exemple quand Louis Jouvet interprétait Tartuffe, les spectateurs avaient l'impression que ce n'était pas Jouvet qui se transformait en Tartuffe, mais Tartuffe qui se transformait en Jouvet. Dans le domaine shakespearien des monstres sacrés tels que Laurence Olivier, John Gielgud, Ralph Richardson, Edith Evans, Peggy Ashcroft, Claire Bloom, ont pu de la même façon investir de leurs caractéristiques reconnaissables et permanentes les rôles qu'ils et elles assumaient, sans que l'auteur ait eu à en souffrir, car le charisme qui émane d'un acteur ou d'une actrice populaire contribue à la puissance et à la crédibilité de l'incarnation, à la mystérieuse osmose qui s'opère entre l'interprète et le personnage. Pour s'identifier à une créature imaginaire, il faut de l'imagination. Les grands acteurs n'en manquent pas. On appelle quelquefois acteurs de composition ceux qui n'occupent pas le haut de l'affiche, et à qui on demande de s'effacer complètement derrière leurs rôles, d'autant plus que les rôles secondaires dans une pièce sont généralement plus typés, dessinés à plus gros traits que les personnages principaux. Mais la distinction peut paraître abusive car tous les acteurs doivent s'adonner à la composition. On peut cependant se demander si ces caractéristiques reconnaissables et permanentes ne sont pas déjà le produit d'une sorte de déguisement. On dit que Louis Jouvet bégayait à la ville. Sur scène il ne bégayait plus, il devenait Jouvet, l'ineffaçable Jouvet. Il absorbait par une sorte d'osmose des rôles dont on aurait pu penser qu'ils n'étaient pas faits pour lui. Mais il arrive aussi qu'un personnage et un 
acteur ou une actrice se rencontrent comme s'ils s'attendaient depuis toujours, prédestinés à une identification mutuelle, tels qu'en euxmêmes enfin le théâtre les change : ainsi Richard Burton en Coriolan, Michael Redgrave en Prospero, ou Claire Bloom en Viola. L'art cinématographique exploite ce phénomène d'une autre façon, car certains acteurs et actrices appréciés du public obtiennent des emplois taillés pour eux. Ce sont les rôles qui viennent à la rencontre de l'acteur, non l'inverse. Du temps où Gary Cooper, Errol Flynn, Clark Gable, Ingrid Bergman ou Ava Gardner figuraient parmi les valeurs sûres du box-office, leurs admirateurs et admiratrices savaient qu'en allant voir leur star préférée, ils et elles le verraient interprétant un personnage à peu près immuable, ou du moins une variation sur un personnage de base. Il n'en va pas tout à fait de même au théâtre, surtout quand il s'agit de l'interprétation des textes classiques. On peut dire cependant que, sur l'écran ou sur la scène, et quels que soient leur statut et le niveau de leur vedettariat, tous les acteurs, même les plus égocentriques, à la manière d'un Sacha Guitry, appartiennent peu ou prou à la catégorie des acteurs de composition, car toujours ils doivent composer un rôle différent, si peu que ce soit, du précédent ou du suivant. Il existe cependant un cas où la théâtralité n'est pas complémentaire de l'identification avec un personnage, mais se substitue à elle, quand des acteurs mettent en pratique, en la poussant à l'extrême, la théorie de la distanciation, et donnent de leur rôle une sorte de lecture abstraite. Si les spectateurs connaissent la pièce ou en devinent les grandes lignes ils peuvent suivre dans leur tête le contrepoint qui s’instaure entre le comportement présumé du personnage et la déclamation du texte.

Il ressort de tout cela que le théâtre possède une vertu pédagogique qu'on ne trouve dans aucun autre art, car le spectateur, même le moins enclin à la réflexion esthétique, se rend compte, chaque fois qu'il voit une de ses vedettes favorites jouer un rôle particulier, qu'il lui a fallu faire preuve d'intuition, de mimétisme, de technique, en bref d'art, pour parvenir au résultat voulu. La réception par le public du travail réalisé sur la scène suppose également une sorte de travail accompli par ledit public, fondé sur une certaine prise de distance, qui se manifeste ouvertement lors des applaudissements ou des huées, lesquelles interrompent le déroulement de l'action. La distanciation est en fait inhérente à l'art théâtral, et il n'est pas toujours nécessaire de 
mettre en œuvre pour cela le lourd et professoral mécanisme prévu par Bertolt Brecht et ses disciples.

Revenons sur la situation la plus fréquente, celle de l'acteur qui monte sur scène pour revêtir une persona et pour se rendre en principe crédible auprès du public, si l'on s'en tient du moins à la conception traditionnelle qui met l'accent sur la mimésis. Il a à sa disposition les ficelles du métier, car c'est exercer une profession que jouer au théâtre. Cette profession, on la pratique plus ou moins bien, et Shakespeare, acteur lui-même, ne manque pas de fournir quelques commentaires sur le talent ou le manque de talent de ses confrères. Dans le sonnet 23 il fait allusion à un acteur inexpérimenté et victime du trac :

As an unperfect actor on the stage

Who with his fear is put beside his part..

On attribue généralement le trac à l'appréhension d'un trou de mémoire, mais ce n'est sans doute pas la seule cause de ce phénomène. La présence intimidante du public, la crainte de ne pas s'identifier complètement au personnage qu'on doit incarner, contribuent sans doute à ce syndrome. Inversement se montrer trop sûr de soi peut produire des conséquences fâcheuses. Dans le célèbre discours de Hamlet aux acteurs, il est question d'un histrion qui joue son rôle avec outrance en utilisant des procédés un peu trop lisibles :

$\mathrm{O}$, it offends me to the soul to hear a robustious periwig-pated fellow tear a passion to tatters, to very rags... (Ham let, III.ii. 8-9)

Bien que tout le monde connaisse ce texte par cœur, il mérite qu'on s'y arrête un peu plus longtemps que sur l'exemple précédent. Tout d'abord l'expression «a robustious periwig-pated fellow 》 résume à elle seule l'attitude sarcastique du locuteur, qui peut-être sert ici de porte-parole à l'auteur. Le mot fellow se réfère à l'homme ordinaire, membre du vulgum pecus, tel qu'il existe en dehors du théâtre, et qui n'arrive pas à se dégager tout à fait de sa gangue habituelle quand il participe à la cérémonie artistique que constitue une représentation théâtrale. La métamorphose doit être complète, sinon tout tombe à plat. Les connotations lourdement somatiques de l'adjectif robustious, quelque peu inattendu, suggèrent peut-être un manque de spiritualité, donc un manque d'art. En poussant l'exégèse jusqu'à ses ultimes conséquences, on peut déduire de cette description satirique qu'un 
acteur qui monte sur la scène ne doit pas se contenter de se transformer provisoirement en quelqu'un d'autre, il doit aussi opérer une transmutation vers le haut, non se contenter d'une permutation horizontale, autrement dit atteindre un certain niveau esthétique de stylisation et de décantation. Quant à periwig-pated, on y voit à l'inverse une allusion à un accessoire théâtral, mais un accessoire trop routinier, trop conventionnel, un peu ridicule, en l'occurrence mieux adapté à la comédie bouffonne qu'à la tragédie. Il faut d'ailleurs reconnaître que si l'outrance peut apparaître comme un défaut, au nom d'une esthétique du ne quid nimis, elle a son utilité dans la comédie satirique, laquelle pratique l'imitation moqueuse poussée jusqu'à la caricature. La suite du texte nous entraîne sur une autre piste, peutêtre vers une digression dont il semble cependant utile d'assumer le risque. Dans «tear a passion to tatters, to very rags » il est question d'un acteur dont les hurlements et les gesticulations détruisent la crédibilité de son incarnation. Les mots tatters et rags, dans leur synonymie insistante, véhiculent le thème du vêtement, leitmotiv de ce congrès. Si l'identification de l'acteur à un personnage ressemble métaphoriquement au port d'un vêtement, et si le vêtement tombe en lambeaux, c'est parce qu'un excès de véhémence va à l'encontre du but recherché. Seulement ce n'est pas exactement d'un personnage qu'il s'agit, mais d'une passion : "tear a passion to tatters, to very rags ». Malgré la célébrité de ce texte, le mot passion n'a peut-être pas reçu de la part des commentateurs toute l'attention qu'il mérite. Il implique que l'acteur n'est pas simplement quelqu'un qui construit un personnage, c'est-à-dire un caractère, figé, gravé une fois pour toutes avec ses propriétés individuelles et ses idiosyncrasies, mais également et peut-être surtout des passions, c'est-à-dire des états affectifs qui concernent toute l'humanité et ne relèvent pas exclusivement d'une typologie, voire d'une typographie, en accord avec l'origine grecque du mot caractère. Certes, le mot passion dans le texte cité, qu'on pourrait traduire par émotion, a surtout le sens de colère et nous savons que selon la sempiternelle théorie des humeurs il existe des gens plus enclins à la colère que d'autres, d'où un lien avec la caractérologie. Il n'en est pas moins vrai que chacun d'entre nous possède une certaine quantité de bile jaune dans son organisme et a bien quelque expérience de la colère, même si nous ne la ressentons pas le jour où nous devons monter sur scène pour en donner le spectacle. On se souvient à ce sujet 
que dans Le paradoxe sur le comédien Diderot défend la thèse selon laquelle un acteur chargé d'exprimer une émotion violente ne doit pas se laisser lui-même emporter par cette émotion, mais doit la maîtriser, la composer avec une froideur professionnelle qui contraste paradoxalement avec la chaleur des sentiments qu'il communique au public. On n'est pas obligé de considérer la théorie de Diderot, qui évoque a contrario le commentaire de Hamlet sur les mauvais acteurs, comme échappant à toute discussion, mais elle a au moins le mérite de rappeler que ce métier ne consiste pas seulement à incarner des personnages, il consiste aussi et surtout à exprimer des passions, des sentiments, des pulsions, des états d'âme dont la plupart sont communs à l'humanité en général, tels que l'amour, la haine, la jalousie, la peur ou l'audace, l'ambition ou le renoncement, le dépit ou la satisfaction, et mille autres affects qui traversent notre vie de façon plus ou moins récurrente ou accidentelle. Au fond tout l'art de l'acteur ou de l'actrice consiste à représenter ce qui est le propre de l'homme, c'est-à-dire éclater de rire ou fondre en larmes. L'incidence de ce constat d'ordre psychologique sur le métier d'acteur ne contredit en rien la nécessité du déguisement, puisque simuler la fureur, la jalousie, la rancune ou toute autre pulsion ressortit à la même tâche professionnelle que celle qui consiste à se mettre à la recherche de Richard III ou à celle d'Harpagon pour mieux les posséder ou se faire posséder par eux. On peut considérer l'ambition et l'avarice comme des traits de caractère, mais ce sont aussi des propensions auxquelles personne n'est complètement étranger. De même qu'il existe dans les sous-sols du théâtre une garde-robe diversifiée et prête à l'emploi, les comédiens se doivent de posséder en eux-mêmes un ensemble de panoplies psychologiques leur permettant de revêtir diverses attitudes. Et cela d'autant plus qu'il leur arrive assez souvent d'opérer sur la scène plusieurs transformations au cours d'un même spectacle, soit parce qu'ils interprètent plusieurs personnages, soit parce que celui qu'ils incarnent passe par diverses phases, y compris des phases de travestissement.

Nous en arrivons donc au deuxième cas, annoncé comme celui du déguisement intra-diégétique, c'est-à-dire celui qu'accomplissent les personnages eux-mêmes quand, dans le cadre de la fiction dramatique, ils assument un changement d'apparence, tout aussi transparent pour le public que les autres types de déguisement. Mais 
s'il ne trompe pas le public, le but est de tromper d'autres personnages. Le camouflage constitue une arme défensive le plus souvent, offensive parfois ; il facilite les diverses formes d'espionnage, comme lorsque Polixenes et Camillo se livrent à une enquête sur les fréquentations de Doricles alias Florizel. Incidemment les changements de noms accompagnent les déguisements, ou se substituent à eux. Si l'on en croit Juliette, et le simple bon sens, le nom que porte chacun de nous n'est qu'un faux-semblant, à plus forte raison si, comme le font tous les fugitifs shakespeariens, on adopte une nouvelle identité onomastique. Ainsi Belarius dans Cymbeline, non content de se cacher dans une caverne du pays de Galles, se fait appeler Morgan, et affuble les deux fils du roi de noms qui les tiennent dans l'ignorance de leur origine. On comprend que de tels procédés fassent si souvent partie des ressorts dramatiques, puisque les conflits et les intrigues qui forment la trame d'une pièce de théâtre ont presque toujours quelque chose de guerrier, de policier, voire de cynégétique. Si en anglais le mot plot signifie à la fois intrigue dramatique et complot, c'est évidemment parce qu'il existe une analogie entre les deux notions. Or les complots de toutes sortes font un grand usage de déguisements, vestimentaires ou autres. Dans Much Ado About Nothing Margaret se prête sans le savoir au complot ourdi par Don John quand elle se montre à une fenêtre revêtue de la robe de sa maitresse. Dans All's Well that Ends Well Parolles se dit victime d'un complot, et il l'est en effet, tombé dans un piège destiné à révéler sa couardise et sa déloyauté, les artisans de cette machination ayant commencé par revêtir des costumes trompeurs et inventer un langage imaginaire. Une imposture en chasse une autre, et c'est pour faire apparaître au grand jour celle de Parolles que ses impitoyables dénonciateurs ont monté leur stratagème. La mystification punitive infligée à Falstaff, lui-même déguisé en chasseur légendaire et cornu, par toute une population déguisée en fées et en lutins à la fin de The Merry Wives of Windsor, appartient au même registre. Ce même Falstaff a été peu de temps avant victime d'un tour joué par le prince Henry dans la première partie d'Henry IV, lorsque ses hommes et lui, déguisés en brigands, lui dérobent le produit de son propre brigandage. Ces exemples montrent aussi que les déguisements au théâtre ont souvent un aspect ludique qui s'ajoute à leur nécessité fonctionnelle. On comprend aussi par là qu'ils appartiennent en premier lieu au répertoire de la comédie. Curieusement la seule comédie de 
Shakespeare où aucun personnage ne se travestit à aucun moment est The Comedy of Errors, mais cela tient certainement au fait que chacun des deux Antipholus et des deux Dromio n'a pas besoin de se déguiser pour produire des quiproquos, puisque le lusus naturae que représente la gémellité les fait ressembler à quelqu'un d'autre. Dans le même ordre d'idées on peut dire que la nuit noire qui sert de cadre au fameux stratagème du lit, the bed-trick, dans All's Well et dans Measure for Measure, reprend à sa façon le thème du déguisement trompeur. Quant à la mutation de Bottom en monstre hybride, elle est indépendante de sa volonté, mais rien n'empêche de l'inclure dans la longue liste des métamorphoses shakespeariennes.

Dans l'œuvre de Shakespeare il y a au moins une trentaine de situations de ce genre, et plus encore si l'on étend la notion de déguisement au-delà des simples travestissements visibles. Par exemple la simulation de la folie par Titus Andronicus ou par Hamlet peut figurer dans la liste, sans parler de tous les imposteurs et simulateurs qui peuplent son théâtre. Pour en revenir au travestissement proprement dit, on remarque au passage qu'il existe sur un point précis une différence entre les deux types de déguisements : alors que sur la scène élisabéthaine, les personnages féminins sont joués par de jeunes garçons, ce qui d'ailleurs provoque un retournement cocasse quand le personnage féminin se travestit en personnage masculin, on ne trouve au contraire parmi les diégèses dramatiques qu'un seul cas de travestissement d'un homme en femme, celui de Falstaff dans The Merry Wives of Windsor, qui ne dure pas longtemps et ne laisse pas un souvenir très marquant. Il est vrai qu'on pourrait ajouter le déguisement de Flute en Thisbé dans A Midsum mer Night's Dream et celui de l'acteur qui interprète la reine dans The Murder of Gonzago, mais il s'agit là de théâtre dans le théâtre, et l'on retombe dans le cas précédent, ou plutôt les deux types de déguisement se combinent. Le cortège involontairement burlesque des Neuf Preux dans Love's Labours Lost relève également de la mise en abyme. Pour revenir à la représentation de Pyrame et Thisbé, qui relève de l'autoparodie, le déguisement de Snout en mur et de Starveling en clair de lune atteint le sommet du burlesque. À propos de mise en abyme on peut se demander si l'inclusion d'un bal masqué dans une pièce de théâtre en constitue une. C'est plutôt la vie mondaine, qui en se livrant à cette pratique, imite le théâtre. Toujours est-il que le bal masqué 
devient sur la scène un lieu et un moment d'intrigues, de rencontres inattendues, d'indiscrétions, de diffusion de fausses nouvelles, de quiproquos. Dans Shakespeare le plus célèbre est celui du bal des Capulets dans Romeo and Juliet, mais il ne faut pas oublier ceux de Much Ado et d'Henry VIII. La mascarade des gentilshommes navarrais, déguisés en Moscovites dans Love's Labours Lost fait partie des travaux amoureux annoncés par le titre, qui tournent court. Les déguisements les plus célèbres et les plus chargés de substance dramatique sont ceux de quelques héroïnes de comédies ou de tragicomédies : Julia, Rosalind, Viola, Imogen, qui pour diverses raisons mais surtout pour se protéger des dangers qui guettent les femmes, se travestissent en garçons. Celia, dans As You Like It, et Helena, dans All's Well That Ends Well se déguisent également, mais sans changer de sexe. Dans tous les cas l'auteur ne se soucie nullement de respecter la vraisemblance, laquelle ne constitue pas un impératif catégorique de l'art théâtral. Ces divers personnages revêtent des déguisements pour se protéger ou pour se livrer à diverses manipulations, et y prennent goût, car ils ou plutôt elles prolongent la supercherie au-delà du nécessaire, mais quel que soit leur motif, elles trompent les autres personnages sans tromper le public, complice du jeu théâtral, et d'autant plus complice qu'il est amené, comme dans le cas précédent de l'acteur qui assume un caractère ou un état d'âme éventuellement étranger ou inhabituel à sa nature, à suivre l'auteur dans ses commentaires métadramatiques et à y ajouter les siens.

Le sommet de la convention et de l'appel à la suspension de l'incrédulité est atteint dans King Lear, quand pour échapper aux poursuites, Edgar se fait passer pour un fou échappé de Bedlam, et se dépouille de ses accoutrements habituels. La nudité comme moyen de ne pas se faire reconnaître assume une signification symbolique : dans un monde de faux-semblants, l'absence de vêtements constitue le plus radical des déguisements, tout comme la sincérité de Cordelia la met à l'écart du jeu social. De même que cette princesse déchue n'est plus reconnue, au sens où reconnaître signifie légitimer, par son père comme étant sa fille, plus personne ne reconnaît au sens propre Edgar à partir du moment où il démasque son corps des attributs signalétiques de son identité mondaine.

Sur ce point le théâtre diffère du roman, non pas des romans contemporains de Shakespeare, comme le prouve le Rosalynd de 
Thomas Lodge, mais des romans d'aventures du XIX ${ }^{\mathrm{e}}$ siècle, dont les auteurs font des efforts laborieux et naïs pour rendre plausible ce qui dans la réalité aurait du mal à y parvenir. Pour prendre un exemple parmi d'autres, lorsque dans The Hound of the Baskervilles Sherlock Holmes adopte un déguisement pour tromper tout le monde, y compris l'ami Watson, Conan Doyle inclut les lecteurs parmi les victimes de la mystification, et ne leur révèle la supercherie qu'au dernier moment. C'est exactement l'inverse qui se passe dans le théâtre de Shakespeare, où les coups de théâtre sous forme d'anagnorisis ne sont pas destinés en premier lieu au public, mais aux personnages trompés par les apparences, comme l'a maintes fois souligné Bertrand Evans ${ }^{1}$. Le public est toujours mis dans le secret, ce qui l'aide à percevoir le mécanisme dramatique, mais il s'ajoute au processus une connivence avec l'auteur qui place ce public au cœur même de la théâtralité. Il a déjà été question de cette connivence, à propos de l'acceptation par le public de la transformation des acteurs en personnages, elle s'exerce aussi quand c'est le personnage qui se transforme tout en restant visuellement à peu près semblable à lui-même. Il a été question précédemment de l'art cinématographique. J'y reviens pour souligner une fois de plus en quoi les conventions de l'écran s'écartent de celles de la scène. Le déguisement s'y pratique assez souvent, mais si en général le public sait à quoi s'en tenir sur l'identité réelle du personnage travesti, car contrairement à ce qui se passe quand on lit un roman, le spectateur d'un film voit la physionomie des acteurs et des actrices, du moins le plus souvent, on constate que le réalisateur et ses assistants font tout ce qui est en leur pouvoir pour donner un semblant de vraisemblance à ce qui n'en a pas vraiment. Quand on assiste à une projection de Some Like It $H_{o t}{ }^{2}$, par exemple, on reconnaît Jack Lemmon et Tony Curtis sous leurs oripeaux usurpés, mais après avoir vu le film on suppute que pendant toute la durée du tournage, les deux malheureux ont dû passer chaque matin et chaque après-midi une heure ou deux dans l'atelier de maquillage, et qu'ils ont peut-être fait appel à des orthophonistes pour apprendre à modifier leur voix. Les déguisements de femmes en hommes, comme celui

\footnotetext{
${ }^{1}$ Shakespeare's Comedies, Oxford, O.U.P., 1960.

${ }^{2}$ Film écrit et réalisé par Billy Wilder en 1959.
} 
d'Andrée Debar dans Le secret du chevalier d'Éon ${ }^{3}$, ou de Julie Andrews dans Victor Victoria ${ }^{4}$, furent effectués avec autant de soin, en évitant les procédés grossiers comme les barbes et moustaches postiches. On n'en demande pas tant au théâtre, car l'illusion comique s'appuie en grande partie sur la collaboration du public, qui est toujours admis, au moins mentalement, à voir l'envers du décor.

Bien entendu le discours moralisateur que suscite le thème des fausses apparences revient souvent. On trouve dans l'œuvre de Shakespeare 46 occurrences du mot disguise et de ses dérivés, le plus souvent assortis de condamnations, comme dans le monologue de Viola dans Twelfth Night :

Disguise, I see thou art a wickedness

Wherein the pregnant enemy does much.

(II.ii.26-7)

« The pregnant enemy » désigne un séducteur perfide, qui peut être aussi le diable, le père des mensonges selon une expression proverbiale qui avait cours du temps de Shakespeare. On peut ajouter à cela les notions que recouvrent des mots comme dissemble, mask, hide, et leurs dérivés, souvent utilisés dans des sens proches de celui de disguise, et dont on trouve dans Shakespeare plus de deux-cents occurrences. Les mots en question sont parfois utilisés ensemble, comme mask et hide dans le sermon contre l'hypocrisie que Brutus s'adresse à lui-même, lorsqu'il voit ses complices s'envelopper le visage pour ne pas être reconnus, même en pleine nuit :

O conspiracy,

Sham'st thou to show thy dang'rous brow by night,

When evils are most free? $\mathrm{O}$ then by day

Where wilt thou find a cavern dark enough

To mask thy monstrous visage? Seek none, conspiracy.

Hide it in smiles and affability: (Julius Caesar, II.i.77-82)

Comme toujours dans Shakespeare on glisse du sens littéral au sens figuré, et vice versa, de sorte que du déguisement de l'apparence physique au déguisement psychologique ou mental la transition s'opère avec douceur. On n'en finirait pas d'énumérer les cas où un personnage pourrait reprendre à son compte la parole énigmatique et pourtant

\footnotetext{
3 Film réalisé en 1959 par Jacqueline Audry sur un scénario de Cecil Saint-Laurent et Pierre Laroche.

${ }^{4}$ Film écrit et réalisé par Blake Edwards en 1982.
} 
parfaitement claire de Iago : «I am not what I am. »(Othello, I.i.65). Les mensonges ont le plus souvent des intentions pernicieuses, mais il existe aussi de pieux mensonges, des tromperies vertueuses, comme celle que Malcolm met en œuvre quand il met Macduff à l'épreuve. Dans tous les cas l'acteur doit faire preuve d'habileté, et comme toujours il doit compter sur la collaboration du public. Par une technique appropriée il doit faire comprendre à celui-ci que le personnage est en train de mentir, mais en même temps donner une apparence de sincérité à ses propos ou à son attitude, afin de rendre plausible le crédit qu'il obtient de ses victimes. Certes le mensonge n'est pas une spécialité exclusivement théâtrale. Nous le rencontrons si souvent dans la vie réelle que sur ce point le dramaturge et ses interprètes n'ont pas à faire de grands efforts pour accomplir la mimésis recommandée par Aristote. Rendre au théâtre la vérité de la vie consiste souvent à rendre l'absence de vérité qui caractérise la vie. Simuler un simulateur, comme le fait tout acteur qui interprète le rôle d'un tartuffe, c'est une tâche presque quotidienne pour les praticiens de ce métier, et tout le monde sait que le mot grec hupokritès signifiait comédien.

Mais la dialectique shakespearienne est habile à faire basculer la thèse dans l'antithèse et dans la synthèse. Si l'on voulait tirer de cette œuvre une philosophie du vêtement, on la trouverait aussi tortueuse et contradictoire que celle de Carlyle dans Sartor Resartus. Certes le vêtement peut symboliser l'imposture, comme lorsque le grossier Cloten s'empare du costume de Posthumus avec l'intention de commettre un odieux attentat contre Imogen, mais ne réussit, une fois décapité, qu'à provoquer un quiproquo pathétique et burlesque, ou comme dans l'invective qu'envoie Kent à Oswald dans King Lear : « A tailor made thee. » (II.ii.55), ce qui signifie que sous le costume il n'y a rien, contrairement à Edgar alias Tom, dans la même pièce, qui sans le moindre costume, devient une sorte de prophète inspiré. Toutefois si la nudité, par sa radicalité même, et son association proverbiale avec la vérité, a pour effet de dévoiler non seulement le corps, mais les ressorts cachés du comportement et les secrets plus ou moins mystiques de l'univers, Shakespeare peut aussi, par un retournement paradoxal, proposer une conception fantastique et visionnaire du vêtement. 
La robe de moine assumée par le duc dans Measure for Measure équivaut pour lui à une sorte d'anneau de Gygès, qui lui donne un don d'invisibilité et d'ubiquité ainsi que la faculté de sonder les reins et les cœurs. Le cynique Lucio s'en tient au proverbe qui dit que l'habit ne fait pas le moine, mais il se trompe, car cet habit fait du duc un supermoine doué d'omniscience et d'omnipotence. Grâce à une sorte de péripétie visuelle et conceptuelle où se plaît le théâtre, le déguisement peut donc avoir un effet paradoxalement révélateur. On croit se cacher, on s'expose aux autres et à soi-même. Dans Le jeu de l'amour et du hasard ou dans She Stoops to Conquer, ou pour revenir à Shakespeare, dans The Taming of the Shrew, où Lucio et Tranio se substituent l'un à l'autre, le travestissement des maîtres en domestiques dévoile les virtualités de leur nature, ou bien suggère que les emplois que la fortune et la société attribuent aux individus pourraient bien être dans la réalité aussi interchangeables que sur la scène d'un théâtre ou comme ils l'étaient jadis au cours des brèves saturnales romaines, ce qui rappelle les liens qui rattachent la comédie shakespearienne à l'esprit du carnaval. Le travestissement peut avoir des effets magiques, comme la robe et les accessoires de Prospero. Les hauts-de-chausses et l'épée au côté que porte Viola alias Cesario ne font pas d'elle un bretteur redoutable, mais il arrive qu'une transformation intérieure résulte d'un changement d'apparence. L'habit noir qui transforme Portia en avocat dans The Merchant of Venice lui confère mystérieusement assez d'astuce juridique pour tirer son client d'un mauvais pas. De même l'habit noir de médecin que revêt Toinette dans Le malade imaginaire infuse en elle assez de science médicale pour déceler l'origine pulmonaire et non imaginaire hélas, du mal dont se meurt à l'instant même l'acteur-auteur qui joue Argan. Quant à l'habit noir de notaire dont s'affuble Despina dans Così fan tutte il lui permet, comme par l'effet d'un antidote homéopathique, de trouver une issue à l'imbroglio que les deux autres déguisements ont créé.

La dénonciation des contrefaçons et des charlatanismes en tous genres ne constitue pas dans Shakespeare une préoccupation aussi obsédante que chez Ben Jonson. La philosophie à la Montaigne qu'on peut deviner derrière son œuvre, ainsi que la manipulation des artifices à laquelle il se livre dans la pratique de son métier, laissent supposer qu'on ne saurait en tirer l'idée simpliste qu'il existe entre les 
apparences et la réalité une différence tellement tranchée qu'elle ne peut échapper qu'aux fous et aux filous. Lui-même, en tant qu'inventeur de fiction - n'oublions pas qu'en français le mot fiction ne désigne pas seulement le genre du roman - passe son temps à feindre, si l'on traduit ainsi le verbe feign - faut-il rappeler qu'il appartient à la même famille que fiction ? - qu'utilise Lorenzo à propos de la création poétique :

Therefore the poet

Did feign that Orpheus drew trees, stones and floods

(The Merchant of Venice, v.i.79-80)

L'illusion contribue aux grands équilibres de la vie. Ne la condamnons pas systématiquement. De l'artificialité des procédés dont vit le théâtre, et qui n'ont pas pour but de tromper le public, mais de l'associer au travail de l'imagination, naît une révélation parfois troublante, non seulement sur la question rebattue de l'apparence et de la réalité, mais sur la théâtralité de la vie quotidienne et du comportement. La mimésis fonctionne dans les deux sens. Le théâtre imite la vie en la stylisant, mais parfois c'est la vie qui imite le théâtre. Dans des espaces autres que celui des planches, les hommes, les femmes, et les enfants, ces derniers manifestant des penchants et des dons histrioniques aussi remarquables que précoces, notamment dans l'art de la singerie parodique, se déguisent et se donnent en spectacle ; par exemple quand deux époux se font ce qu'on appelle une scène, adoptent des postures ostentatoires, cultivent une rhétorique qui déguise la pensée ou les sentiments, et cherche à agir sur les destinataires. Il ne faut cependant pas calomnier la rhétorique, elle ne consiste pas toujours à donner un visage spécieux au mensonge. Elle tend à l'exagération, à l'extériorisation, à la mise en forme des émotions et des convictions, autrement dit à la théâtralisation du discours. Le théâtre reflète en retour cette réalité. On trouve de nombreux exemples de ce genre d'enflure psychologique et verbale dans les drames historiques et politiques de Shakespeare, et dans beaucoup d'autres pièces, où les déclamateurs de tirades véhémentes cherchent à se convaincre euxmêmes autant qu'à convaincre leurs auditeurs ; par la même occasion ils se fabriquent une essence à base de mauvaise foi, comme dirait Sartre, un personnage bien dessiné, brillamment coloré, capable de se mettre en valeur auprès d'autrui ainsi qu'à leurs propres yeux. Transposés dans la réalité, d'où ils viennent, un Richard II, un Falstaff, 
un Hotspur, ressembleraient déjà à des personnages de théâtre. Les personnes que nous rencontrons autour de nous ou devant notre miroir sont parfois des personnes en effet, personae au sens étymologique et théâtral: elles portent un masque, ou plusieurs masques plus ou moins grimaçants au cours de leur existence ou au cours de la même journée. Nous sommes assez souvent des comédiens sans le savoir, comme dit Balzac, mais parfois aussi des comédiens conscients du jeu auquel nous nous livrons, y compris quand nous jouons à être nous-mêmes, et cumulons les deux fonctions inséparables que sont celle de l'acteur et celle du spectateur. Dans sa célèbre tirade d'As You Like It, Jaques attribue aux hommes et aux femmes, sur ce grand théâtre du monde, sept rôles successifs au long de leur vie. En réalité chacun de nous en joue beaucoup plus de sept, si ce concept de rôle englobe toutes les attitudes que nous assumons. On a même vu, au cours par exemple d'un congrès de spécialistes, des individus qui bien que n'ayant rien d'original à communiquer, se déguisaient en auteurs de communications, montaient à la tribune pour parader, simulaient la modestie contrite pendant la cérémonie des éloges prononcés par le président de séance, prenaient la parole et la gardaient pendant une demi-heure et parfois plus. Eh bien je crois que la demi-heure est écoulée, il me reste à vous remercier de votre attention et à prendre congé. 